\section{Cooperation in rats without prior individual training}

\author{
MILTON E. ROSENBAUM and STEPHEN W. EPLEY \\ University of Iowa, Iowa City, Iowa 52240
}

Pairs of rats were placed in a mixed-motive situation in which they were dependent upon each other in order to avoid electric shock and obtain food. The results show that rats are capable of establishing behavioral interdependence in the absence of individual training to perform certain components of the interdependent behavior.

In the few reported laboratory demonstrations of effective cooperation in subhuman species, training has been conducted of each individual in the component responses necessary for the later execution of the cooperative behavior. In the present study, the development of cooperative behavior in rats was examined in the absence of individual training.

The procedure employed in the present study was adapted from two studies by Daniel $(1942,1943)$, in which he demonstrated that two rats, when placed in an apparatus in which they were dependent upon each other, were capable of cooperatively solving the rather difficult problem with which they were faced. In this situation, the food was available from a dish placed on an apparatus floor made of grid bars through which electric shock was delivered. A platform was located at the end of the apparatus, which allowed escape from the shock, but at a distance too great to permit access to the food dish without leaving the platform. The animals placed in this environment were faced with a difficult, and potentially unsolvable, double-motive situation provided by the incompatibility of feeding and avoiding shock. Their survival was at stake, since food was available only in the apparatus. The problem could be solved because, when the platform was occupied by a rat, microswitch contacts were made that eliminated shock. When two animals were placed into the apparatus, one could perch on the platform while the other could feed in the absence of shock. Daniel demonstrated that the rats developed a pattern of interchange from platform to food and back which allowed both animals of a pair to avoid shock and to obtain sufficient food.

Despite frequent allusions to Daniel's studies in the intervening years as one of the few instances of true interdependent action on the part of animals, no replications or extensions have been reported. The major purpose of the present study, in addition to replication, was to add a feature to the experiment that is a significant departure from Daniel's animals were trained independently to differentiate between the conditions and responses appropriate to the food and shock conditions. Over an initial series of days, each rat alone experienced the food in the absence of any shock. Next, the animals were trained to escape the electrified grid by going to the platform with no food present. Then trials with food alone and shock alone were intermixed. Only when the discriminative behaviors of feeding in the absence of shock and ascending the platform in the presence of shock were well established were the animals placed in the apparatus together.

It can be seen that Daniel's procedure was designed to provide each animal with the component stimulus-response connections necessary for problem solution before the members of a pair were exposed to each other. Problem "solution was effected by coordination of these well-established repertoires.

The procedures of the present study were directed toward exploration of the possibility that cooperative responding would occur when the members of a pair did not receive individual training prior to formation of the pair and exposure to their interdependency problem.

Almost all features of the present study were similar to Daniel's procedure, except that no training was given in escaping the electrified grid. After 4 days of experience with the food on the part of each animal alone, two animals were dropped onto the electrified grid and experienced shock for the first time.

\section{APPARATUS}

The apparatus consisted of a $20.3 \times 47 \mathrm{~cm}$ box formed by joining two rodent test cages (Scientific Prototype, Model A100) after one wall had been removed from each cage. A Masonite platform, $19.7 \times 12.7 \mathrm{~cm}$, extended across one end of the box and was mounted on microswitches which served to eliminate shock if any part of an animal depressed the procedure. In Daniel's procedure, the platform. The remaining floor area of the box consisted of grid bars on which a glass food dish was located $23 \mathrm{~cm}$ from the platform. This distance rendered it impossible for an animal to reach the food dish without stepping off the platform; however, tactual contact was possible between a rat feeding at the dish and a rat that maintained one or both rear feet on the platform. Shock was delivered through a scrambled shock source (Scientific Prototype, Model 4008-J) at an intensity of .56 mills through a 44-K resistor, as measured at the direct output terminal of the source. The food was composed of wet Purina Rat Chow made into a mash of five parts food to six parts water.

PROCEDURE

Ten female Sprague-Dawley albino rats, all between 100 and 115 days old, were housed separately and were first given habituation individually to the apparatus for 5 min each day for 4 days. During this period they were fed $1 \mathrm{~h}$ each day in their home cage, and, throughout the study, water was available on an ad lib basis in the home cage. On the next 4 days the animals were provided food in the apparatus only, and each day each rat alone experienced two $7.5 \cdot \mathrm{min}$ trials of food availability with a $10-\mathrm{sec}$ interval between trials. Five pairs were constituted randomly, and for the next 19 days, the following procedure was employed: Both members of a pair were placed simultaneously into the apparatus directly onto the electrified grid. They were left in the apparatus for $105 \mathrm{sec}$ and then removed for an intertrial interval of $25 \mathrm{sec}$, during which they were held passively. Twelve such trials were conducted each day for each pair. For each animal, presence on the platform or at the food dish was recorded by means of a four-channel pen recorder activated by four switch buttons which were operated by the $E$.

RESULTS AND DISCUSSION

The major behavioral measure was the number of interchanges executed by each pair of animals. An interchange was tabulated if at one point one animal was on the platform and the other at the food dish, and these positionings were followed by a reversal of location. What is differentiated in the upper left of Fig. 1 is the mean number of interchanges effected by the five pairs with or without shock; that is, some interchanges occurred as a function of one animal stepping off the platform before his partner had returned (with shock), while a clearly effective interchange was one in which an animal delayed stepping off the platform until his partner had returned to it (without shock). The number of 

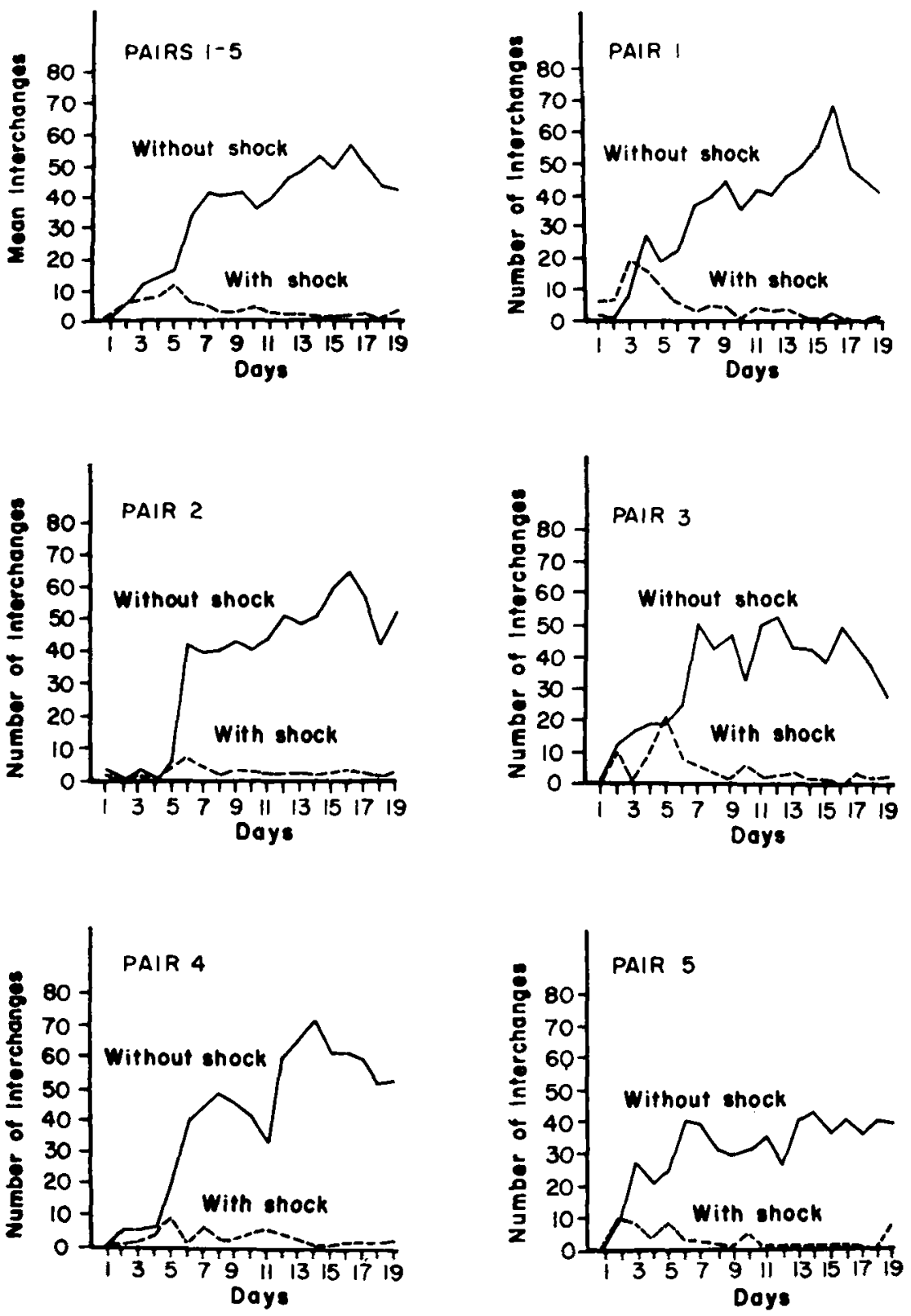

Fig. 1. Number of interchanges between platform and food executed by pairs of rats over days. Differentiation of interchanges is made on the basis of execution without shock (when both animals are at least momentarily on the platform together) and with shock (when both animals are at least momentarily on the electrified grid together). Combination of the curves for with and without shock yields total interchanges.

interchanges in the early days of the study were few, and those that did occur were frequently with shock. Within a few days it was clear that the number of interchanges was increasing, and, in addition, they were for the most part without shock. By the last 5 days a sizable number of interchanges were occurring, and less than $3 \%$ of them were with shock. The increase in mean total interchanges from the first 5 days to the last 5 days is significant $(t=4.44, p<.02)$. The number of interchanges performed by each pair is presented also in Fig. 1.

An indication of the degree to which animals were solving the problem they faced is reflected in whether or not they were receiving the sustenance they required. The weight of each animal was recorded after each day's series of trials, and mean weight in grams is presented in Fig. 2. During the initial days, there was a continuous weight drop which reached its maximum on Days 7 and 9 . At this point, weight gain started and continued to the end of the study, when it reached almost the level of the start of the study. A comparison of the two figures indicates that the weight increase began at approximately the time when the animals were making a sizable number of interchanges.

Although the above data demonstrate the development of the solution to the survival problem with which the animals were faced, the details of the process are quite complex. By no means did the animals spend the available time racing back and forth between the platform and the food. In Fig. 3 the mean proportion of the time during which (1) both animals were on the platform together, (2) one animal was at the food dish alone and the other was on the platform, and (3) both animals were on the grid together (receiving shock) is presented. The small percentage of time not accounted for in Fig. 3 constitutes primarily those occasions during which one animal was on the platform and the other animal was on the grid bars but not at the food dish. This relationship appeared most often during the later trials of a day's series when the animals were apparently satiated. It may be seen that there is a continuous decrease in time together on the grid. Time eating and on the platform together were approximately equal and remained relatively stable over the course of the study. During the early days, little food, if any, was obtained by most of the animals during approaches to the food dish. Although a single rat frequently spent a relatively lengthy time in proximity to the food dish, apprehension was clearly manifested and the rat commonly returned to the platform without food.

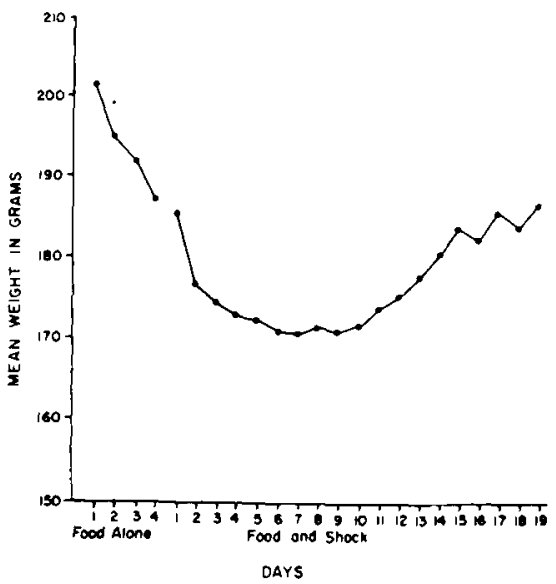

Fig. 2. Mean weight in grams during exposure individually to food in the apparatus and during exposure to food and shock when mebers of each pair were placed in the apparatus together. 


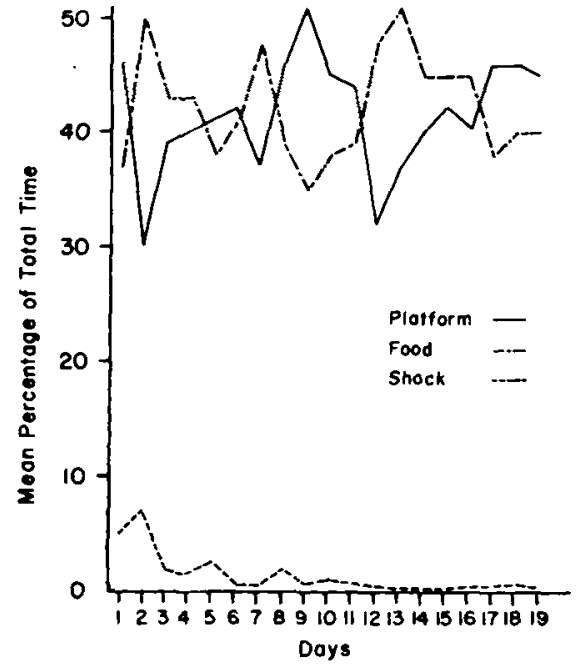

Fig. 3. Mean proportion of time over days during which both members of a pair were on the platform together and on the electrified grid together and the mean proportion of time during which one animal of a pair was at the food dish alone.

In most pairs, after a period of interchange development, one member began to carry food back to the platform in a gathered mass and consume it there. This allowed the other member to feed at the dish until the food carrier was ready to step off the platform to gather more food. This pattern of interchange in some instances appeared to be based on relatively more independent action on the part of the food carrier and somewhat more dependent responding by the other member of the pair. However, the food carrier evidenced sensitivity to its partner by poising to step off the platform when it had finished eating but delaying until its partner had returned. Execution of interchanges were often very precise, with one rat stepping off the platform only when the other rat made one foot contact with it. The few interchanges with shock in the later stages of the study were, for the most part, based on momentarily faulty executions, as when a rat on the platform left it just before the other rat returned.

Observation of the animals suggested that a variety of cues served to implement the interchange behavior. Tactual contact between an animal on the platform and one on the grid was frequent. As Daniel had observed, a rat on the platform would reach off, holding one foot on the platform, and nudge the feeding rat. At times, with both rear feet remaining on the platform, a rat would climb onto the back of its partner. Movements of the rat on the platform involved in poising to step off seemed to serve as an auditory cue eliciting return to the platform by the feeding rat. Many of the animals, when at the food dish, oriented for visual reception of any tendency of their partners to step off the platform, and they responded sensitively to such cues.

Although it is unlikely as a full explanation of Daniel's results, it is possible that his pretrained rats learned, when alone, the appropriate behavior of ascending the platform in the presence of shock and going to food in its absence (a "hot"-platform, "cold"-food discrimination), and, therefore, when paired in the apparatus, each member was then well on the way to producing the ingredients for effective interchange behavior. In the present study, the 4 days of preexposure to food in the apparatus when alone can be viewed merely as "informing" the animals of the availability of the food. First exposure to the shock, to the other animal, and to the function of the platform in providing escape as well as termination of shock occurred essentially simultaneously. The development of the interchange pattern was based on adjustments and learning while in the interdependent relationship and was not related to prior discriminative experience. Previous considerations of cooperative behavior have emphasized the necessity for pretraining of the requisite responses in order for cooperation to be demonstrated in lower forms (Keller \& Schoenfeld, 1950).

The treatment of the observed behavior as cooperative is not based on traditional features such as coordination of responding when the same response is demanded of two organisms at the same time or in the type of behavior involving intent to assist another. Cooperation is treated here as characterized by the mutual reinforcement contingencies resulting from the behavior of each member of the pair (Rosenberg, 1960; Zajonc, 1969).

At the conclusion of the study reported above, two exploratory procedural changes were introduced. On the first day following the completion of the study, the membership of four of the five pairs of rats was reconstituted. For example, Rat A of Pair 1 was placed in the apparatus with Rat $C$ of Pair 2, and Rat B of Pair 1 and Rat D of Pair 2 were placed in the apparatus together. Although in the early trials for that day some decrement in interchange behavior was noted, the mean number of interchanges (with and without shock) was approximately at the level performed on the previous day. (The mean total interchanges were 50.5 .)

On the next 8 days, original pairings were reinstated and shock was either totally or partially eliminated. For two of the five pairs, the shock was on for the first $10 \mathrm{sec}$ of each trial and then turned off while the remaining pairs were placed on a nonelectrified grid at the start of each trial. Interchanges continued to be performed in both groups but with decreasing frequency. The pairs of animals exposed to total cessation of shock dropped from a level of 45.2 mean total interchanges on the first 2 days to 23.3 mean interchanges on the last 2 days. The corresponding values for the pairs receiving shock for $10 \mathrm{sec}$ was 53.6 to 29.6. As was expected, there was an increasing tendency in both groups for both members of a pair to remain on the grid together for increasing lengths of time. These exploratory procedures represent initial attempts to consider variables affecting the transfer and maintenance of cooperative behavior in rats.

\section{REFERENCES}

DANIEL, W. J. Cooperative problem solving in rats. Journal of Comparative Psychology, 1942, 34, 361-368.

DANIEL, W. J. Higher order cooperative problem solving in rats. Joumal of Comparative Psychology, 1943, 35, 297-305.

KELLER, F. S., \& SCHOENFELD, W. N. Principles of psychology. New York: Appleton-Century-Crofts, 1950.

ROSENBERG, $S$. Cooperative behavior in dyads as a function of reinforcement parameters. Journal of Abnormal \& Social Psychology, 1960, 60, 318-333.

ZAJONC, R. B. Animal social psychology: $A$ reader of experimental studies. New York: Wiley. 1969. 